\title{
ESTUDO DA RAZÃO MOLAR GLICEROL/ÁGUA E DO EFEITO DA TEMPERATURA NA REAÇÃO DE GASEIFICAÇÃO DO GLICEROL EM ÁGUA SUPERCRÍTICA
}

\author{
T. V. BARROS ${ }^{1}$, A. D. S. VIDOTTI ${ }^{1}$, R. GUIRARDELLO ${ }^{2}$ e A. C. D. FREITAS ${ }^{1}$ \\ ${ }^{1}$ Universidade Federal do Maranhão, Coordenação do curso de Engenharia Química \\ ${ }^{2}$ Universidade Estadual de Campinas, Faculdade de Engenharia Química \\ E-mail para contato: thiago1390@gmail.com; ads.vidotti@ufma.br; guira@feq.unicamp.br; \\ acd.freitas@ufma.br
}

\begin{abstract}
RESUMO - A busca por novas fontes de energia que substituam os combustíveis fósseis é crescente. O biodiesel aparece como alternativa por ser um combustível biodegradável e não-tóxico. O glicerol é considerado um excedente na produção de biodiesel e uma opção interessante é o uso desta substância na gaseificação com água supercrítica, gerando diversos compostos, entre estes, o hidrogênio. A SCWG do glicerol foi analisada através da metodologia de minimização da energia de Gibbs, identificando-se como um problema de otimização não-linear. As simulações foram realizadas no software GAMS ${ }^{\circledR}$ com auxílio do solver CONOPT3 e observou-se que a produção de hidrogênio é favorecida a altas temperaturas e baixas composições molares de glicerol. Em particular, a melhor condição encontrada para este fim foi observada a $1200 \mathrm{~K}$ e proporção molar de 1/60 (mol glicerol/mol água). Os resultados também apresentam a formação de outras substâncias de interesse industrial: ácido acético, etano, etanol, metano, metanol e propano, cujas concentrações permaneceram baixas em todas as condições estudadas. Na proporção molar de $1 / 60$ a $1200 \mathrm{~K}$, os componentes mencionados somam frações molares inferiores a $0,03 \%$.
\end{abstract}

\section{INTRODUÇÃO}

A demanda energética mundial tem crescido nos últimos anos. Os combustíveis fósseis têm sido amplamente utilizados como fonte de energia. Todavia, são considerados poluentes do meio ambiente e por isto motivam relevante preocupação ambiental. Torna-se necessário a descoberta de novas alternativas que possam suprir ou complementar a matriz energética global.

Entre as possíveis fontes de energia, surge o biodiesel, que é um combustível biodegradável, não tóxico e pouco poluente, produzido a partir de óleos vegetais ou gorduras animais. Vários subprodutos são obtidos em sua preparação sendo um destes o glicerol. Segundo Schwengber et. al. (2016) no ano de 2013, cerca de 350 mil toneladas de glicerol foram produzidas em plantas de biodiesel no Brasil. Com o aumento da produção, é esperado que sejam encontrados novos usos para o excesso de glicerol presente na fabricação.

Diferentes métodos vêm sendo testados para o processamento do glicerol bruto, entre eles a reforma a vapor, reforma oxidativa, reforma autotérmica, etc. Nos últimos anos, uma técnica 
que vem sendo explorada é a gaseificação deste com água supercrítica. A gaseificação oferece uma alta flexibilidade no uso de diferentes tipos de alimentação bem como na geração de diferentes produtos. Através da gaseificação, o glicerol pode ser convertido em gás de síntese e além dos produtos principais, pode gerar vários outros subprodutos tais como: propano, etano, aldeídos, etc. (Rémon et. al., 2016). Entre esses processos, a reação de gaseificação em água supercrítica vem ocupando um papel de destaque, devido as elevadas taxas de produção de $\mathrm{H}_{2}$ obtidas no processo, conforme mostrado no trabalho de Freitas e Guirardello (2014).

\subsection{Gaseificação com água supercrítica}

A literatura sobre gaseificação de hidrocarbonetos em água supercrítica vem aumentando nos últimos anos, embora a maioria foque nos parâmetros operacionais, com poucos estudos envolvendo modelos cinéticos e quantitativos.

As principais vantagens deste processo estão relacionadas às propriedades únicas que a molécula de água adquire em estado supercrítico, tais como as mudanças em sua constante dielétrica, capacidade calorífica, etc. possibilitando que ela possa ser dissolvida em um solvente não polar ou dissolver os mesmos (Guo et. al., 2010).

O mecanismo da SCWG (Supercritical Water Gasification) do glicerol ainda não foi completamente elucidado pois diversos intermediários são formados durante a reação. O vapor resultante da reação é uma mistura de gases e o conteúdo de $\mathrm{H}_{2}$ depende da temperatura e da razão molar de glicerol $/ \mathrm{H}_{2} \mathrm{O}$ presente na alimentação (Matsumura et. al., 2005). Em sua maioria, estes intermediários se decompõem para formar $\mathrm{CO}, \mathrm{CO}_{2}$ e $\mathrm{H}_{2}$. A produção de hidrogênio pode ser favorecida neste caso com alta concentração de água que favorece a reação water-gas shift (Equação 2), produzindo mais $\mathrm{CO}_{2}$ e $\mathrm{H}_{2}$. O esquema geral de possíveis reações para formação dos principais produtos da SCWG do glicerol é apresentado nas Equações 1 e 2 abaixo:

$$
\begin{aligned}
& \mathrm{C}_{3} \mathrm{H}_{8} \mathrm{O}_{3}+\mathrm{H}_{2} \mathrm{O} \rightarrow 3 \mathrm{CO}+4 \mathrm{H}_{2} \\
& \mathrm{CO}+\mathrm{H}_{2} \mathrm{O} \leftrightarrow \mathrm{CO}_{2}+\mathrm{H}_{2}
\end{aligned}
$$

Entre as pesquisas de diversos autores, observa-se que o interesse na produção de hidrogênio a partir da gaseificação de hidrocarbonetos é crescente. $\mathrm{O} \mathrm{H}_{2}$ gerado na reação produz energia para geração de potência, transporte e outras aplicações, como as células a combustível.

Dentre as diversas análises realizadas no tema, grande parte é focada em avaliar modelos com presença ou ausência de catalisadores e seus diferentes aparatos experimentais (Markočič et. al., 2013). No entanto, alguns autores apresentam modelos termodinâmicos para o processo, que são uteis à medida que são capazes de predizer dados experimentais como guia para design de processos e posteriores avanços. Os dados obtidos são capazes de prever com certa precisão os sistemas reais uma vez que as composições experimentais são bem próximas das composições de equilíbrio, conforme é apresentado no trabalho de Tang e Kitagawa (2005).

Duas abordagens gerais para modelar estes sistemas são propostas: a estequiométrica e a não estequiométrica. 
A estequiométrica baseia-se na premissa de que é conhecido o número de reações possíveis para determinadas espécies do sistema. Portanto, é necessária a descrição detalhada de todas as prováveis reações, o que nem sempre é possível. Na abordagem não estequiométrica não é preciso definir as reações que ocorrem no sistema, apenas o número de possíveis espécies presentes, cujas composições são determinadas a partir da minimização da energia de Gibbs.

O objetivo deste trabalho é, portanto, aplicar a metodologia da minimização da energia de Gibbs afim de avaliar as melhores condições para produção de hidrogênio e identificar as implicações determinadas pela variação da temperatura e da proporção molar entre glicerol e água, verificando também a formação de diferentes compostos de menor relevância ao longo da reação.

\section{METODOLOGIA}

\subsection{Minimização de energia de Gibbs}

A metodologia utilizada para o sistema em estudo no presente trabalho é baseada no critério de minimização da energia de Gibbs em condições de pressão e temperatura constantes, que pode ser formulado como um problema de otimização não linear. Como condição suficiente para garantir o equilíbrio termodinâmico, tem-se que:

$$
(d G)_{T, P} \leq 0
$$

Assim, o problema de otimização através da minimização da energia de Gibbs considerando equilíbrio químico e de fases em um sistema com múltiplos componentes, presentes em três potenciais fases (gás, líquido e sólido) pode ser essencialmente representado pela Equação 4:

$$
\min G=\sum_{J=1}^{N C} \mu_{J}^{g} n_{J}^{g}+\sum_{J=1}^{N C} \mu_{J}^{l} n_{J}^{l}+\sum_{J=1}^{N C} \mu_{J}^{s} n_{J}^{s}
$$

Imposto as restrições de não negatividade do número de mols (Equação 5) e o balanço de átomos (Equação 6):

$$
\begin{aligned}
& n_{i, k} \geq 0 \\
& \sum_{i=1}^{N C} a_{m i}\left(\sum_{k=1}^{N C} n_{i k}\right)=\sum_{i=1}^{N C} a_{m i} n_{i 0} ; m=1, \ldots, N E
\end{aligned}
$$

Onde $a_{\text {mi }}$ é o número de átomos do elemento i no componente m e NE é o número de elementos que possuem o átomo em sua composição. NC representa o número de componentes.

Neste trabalho o sistema foi considerado ideal e consideraram-se até 18 possíveis componentes presentes no meio reacional, sendo água e glicerol como reagentes e os demais: hidrogênio, monóxido de carbono, dióxido de carbono, oxigênio, metano, metanol, etanol, etano, propano, butano, ácido acético, ácido fórmico, nitrogênio, monóxido de nitrogênio, dióxido de nitrogênio e amônia. A pressão foi mantida constante em 320 bar. 
Para resolver as equações acima descritas, foram realizadas simulações no software GAMS $^{\circledR}$ versão 23.9 .5 com auxílio do solver CONOPT3. A descrição deste software pode ser encontrada em Brooke (1998). O modelo foi construído a partir de propriedades termodinâmicas obtidas de Poling et. al. (2001).

\section{RESULTADOS E DISCUSSÃO}

\subsection{Efeito da razão molar glicerol/água}

Afim de avaliar o efeito da razão molar entre glicerol e água, realizaram-se diversas simulações utilizando as proporções de 1/5, 1/20, 1/30, 1/40, 1/50 e 1/60, mol glicerol/mol água, na alimentação.

A Figura 1 mostra as variações de número de mols dos diversos componentes produzidos em função da temperatura para as proporções de 1/5 e 1/60. É possível notar que à medida que se aumenta a concentração de água no sistema, ocorre também um acréscimo da quantidade de hidrogênio produzido, em função do excesso de água, deslocando o equilíbrio da reação na Equação 2 no sentido de produção de $\mathrm{H}_{2}$. Na Figura 1, os dados referentes a água foram suprimidos devido ao excesso desse componente na alimentação.

Figura 1 - Número de mols vs Temperatura da reação: 1/5 (A) e 1/60 (B)

(A)

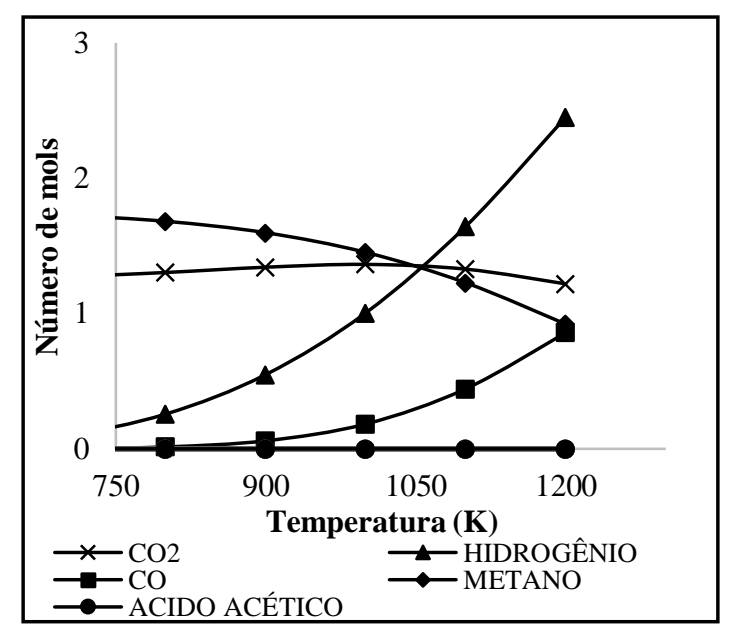

(B)

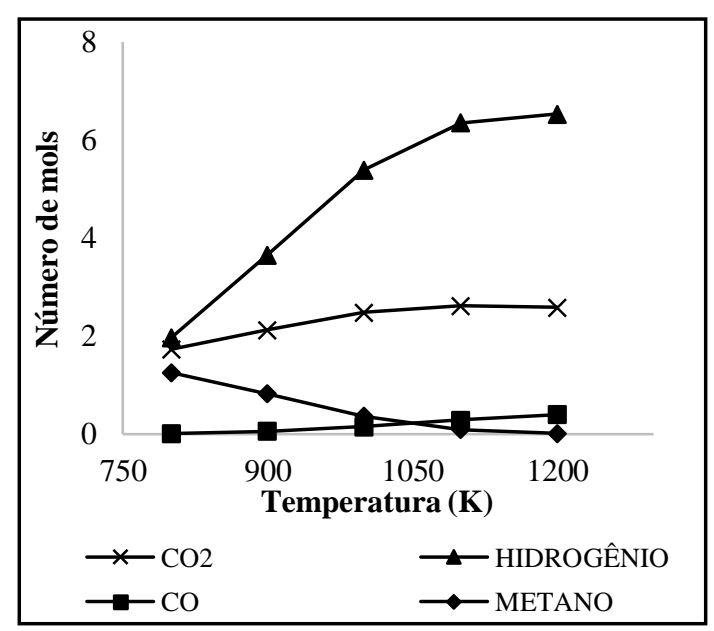

Observa-se também a formação de compostos intermediários no meio reacional. Na proporção 1/60 formam-se: metano, metanol, etano e ácido acético, porém em número de mol muito baixos (com valores máximos ao longo da reação de 1,$26 ; 2 \cdot 10^{-5} ; 1 \cdot 10^{-5}$ e 4,69.10 $0^{-5}$ mols respectivamente). Nesta condição, na temperatura de $1200 \mathrm{~K}$ as frações molares destes componentes representaram valores menores que $0,03 \%$. As composições de metano e etano neste caso decrescem conforme a temperatura de reação aumenta.

Em relação a proporção 1/5 nota-se a formação de outros compostos. Além dos citados anteriormente, formam-se também etanol e propano. Ambos são formados em proporções muito baixas, conforme os dados apresentados na Tabela 1 . 
Tabela 1 - Número de mols formados por componente em função da temperatura. Proporção de 1/5 (mol glicerol/mol de água)

\begin{tabular}{|c|c|c|c|c|c|}
\hline & \multicolumn{5}{|c|}{ Temperatura (K) } \\
\hline Componente & $\mathbf{8 0 0}$ & $\mathbf{9 0 0}$ & $\mathbf{1 0 0 0}$ & $\mathbf{1 1 0 0}$ & $\mathbf{1 2 0 0}$ \\
\hline Ácido acético & $2,2 \mathrm{E}-07$ & $4,1 \mathrm{E}-07$ & $6,2 \mathrm{E}-07$ & $7,6 \mathrm{E}-07$ & $7,2 \mathrm{E}-07$ \\
\hline Água & $5,4 \mathrm{E}+00$ & $5,3 \mathrm{E}+00$ & $5,1 \mathrm{E}+00$ & $4,9 \mathrm{E}+00$ & $4,7 \mathrm{E}+00$ \\
\hline $\mathrm{CO}_{2}$ & $1,3 \mathrm{E}+00$ & $1,3 \mathrm{E}+00$ & $1,4 \mathrm{E}+00$ & $1,3 \mathrm{E}+00$ & $1,2 \mathrm{E}+00$ \\
\hline $\mathrm{CO}$ & $1,4 \mathrm{E}-02$ & $6,0 \mathrm{E}-02$ & $1,8 \mathrm{E}-01$ & $4,4 \mathrm{E}-01$ & $8,6 \mathrm{E}-01$ \\
\hline Etanol & $0,0 \mathrm{E}+00$ & $0,0 \mathrm{E}+00$ & $2,5 \mathrm{E}-08$ & $4,5 \mathrm{E}-08$ & $5,4 \mathrm{E}-08$ \\
\hline Etano & $1,9 \mathrm{E}-04$ & $2,7 \mathrm{E}-04$ & $3,2 \mathrm{E}-04$ & $3,2 \mathrm{E}-04$ & $2,4 \mathrm{E}-04$ \\
\hline Hidrogênio & $2,6 \mathrm{E}-01$ & $5,5 \mathrm{E}-01$ & $1,0 \mathrm{E}+00$ & $1,6 \mathrm{E}+00$ & $2,5 \mathrm{E}+00$ \\
\hline Metano & $1,7 \mathrm{E}+00$ & $1,6 \mathrm{E}+00$ & $1,5 \mathrm{E}+00$ & $1,2 \mathrm{E}+00$ & $9,2 \mathrm{E}-01$ \\
\hline Metanol & $4,3 \mathrm{E}-06$ & $1,0 \mathrm{E}-05$ & $3,0 \mathrm{E}-05$ & $6,0 \mathrm{E}-05$ & $9,0 \mathrm{E}-05$ \\
\hline Propano & $7,9 \mathrm{E}-08$ & $1,0 \mathrm{E}-07$ & $9,3 \mathrm{E}-08$ & $5,3 \mathrm{E}-08$ & $1,5 \mathrm{E}-08$ \\
\hline
\end{tabular}

À medida que a solução de glicerol/água se torna mais diluída (aumentando a concentração de água), observa-se um aumento considerável na quantidade de $\mathrm{H}_{2}$ produzido. Da mesma maneira, o incremento de temperatura resulta em maior conteúdo de hidrogênio. A Tabela 2 apresenta estas informações.

Tabela 2 - Número de mols de Hidrogênio formado em função da razão molar glicerol/água

\begin{tabular}{|c|c|c|c|c|c|c|}
\hline \multicolumn{7}{|c|}{ Número de mols de Hzão formado } \\
\hline & \multicolumn{7}{|c|}{ Razor Glicerol/Água } \\
\hline Temperatura (K) & $\mathbf{1 / 5}$ & $\mathbf{1 / 2 0}$ & $\mathbf{1 / 3 0}$ & $\mathbf{1 / 4 0}$ & $\mathbf{1 / 5 0}$ & $\mathbf{1 / 6 0}$ \\
\hline 800 & 0,26 & 0,77 & 1,09 & 1,39 & 1,69 & 1,96 \\
\hline 900 & 0,55 & 1,57 & 2,17 & 2,72 & 3,21 & 3,66 \\
\hline 1000 & 1,01 & 2,69 & 3,58 & 4,32 & 4,91 & 5,39 \\
\hline 1100 & 1,64 & 3,97 & 4,98 & 5,65 & 6,08 & 6,35 \\
\hline 1200 & 2,45 & 5,06 & 5,83 & 6,21 & 6,42 & 6,53 \\
\hline
\end{tabular}

Com base nos resultados obtidos pode-se evidenciar que os efeitos termodinâmicos foram responsáveis por elevar a produção de $\mathrm{H}_{2}$ nas seguintes condições: temperaturas elevadas e maiores frações molares de água na alimentação.

\section{CONCLUSÃO}

A simulação da reação de gaseificação de glicerol em água supercrítica foi realizada utilizando um modelo termodinâmico formulado como uma minimização da energia de Gibbs global do sistema. Observou-se que a razão molar glicerol/água utilizada para a reação influencia no número de mols de hidrogênio produzido. Soluções mais diluídas de glicerol apresentaram maiores quantidades de hidrogênio formado (1/40, 1/50 e 1/60). A proporção de mistura dos reagentes também ocasiona a formação de compostos intermediários distintos, que foram evidenciados em maiores quantidades em soluções com menores concentrações de água (como exemplo, a solução 1/5). A elevação da temperatura também representou um efeito significativo no aumento do teor de hidrogênio formado, podendo ser considerado um efeito ainda mais pronunciado que o da composição. A produção de hidrogênio aumentou em pelo 
menos $111 \%$ em relação a temperatura de $800 \mathrm{~K}$ e proporção $1 / 5$. Quanto a proporção $1 / 60$ e mesma temperatura, o acréscimo percentual foi de pelo menos $86 \%$.

\section{REFERENCIAS}

BROOKE A., KENDRICK, D., MEEARUS A., RAMAN, R. GAMS - A User's Manual. Washington, DC: In GAMS Development Corp., 1998.

FREITAS, A. C. D.; GUIRARDELLO, R. Comparison of several glycerol reforming methods for hydrogen and syngas production using Gibbs energy minimization. Int. J. Hydrogen Energy, v. 39, p. 17969-17984, 2014.

GUO, Y.; WANG, S. Z.; XU, D. H.; GONG, Y. M.; MA, H. H.; TANG, X. Y. Review of catalytic supercritical water gasification for hydrogen production from biomass. Renew. Sust. Energy Rev., v. 14, p. 334-343, 2010.

MARKOČIČ, E.; KRAMBERGER, B.; BENNEKOM, J. G. V.; HEERES, H. J.; VOS, J.; KNEZ, Z. Glycerol reforming in supercritical water; a short review. Renew. Sust. Energy, v. 23, p. 40-48, 2013.

MATSUMURA, Y.; MINOWA, T.; POTIC, B.; KERSTEN, S. R. A.; PRINS, W.; SWAAIJ, W. P. M. V.; BELD, B. V. D.; ELLIOTT, D. C.; NEUENSCHWANDER, G. G.; KRUSE, A.; JR, M. J. A. Biomass gasification in near- and super-critical water: Status and prospects. Biomass and Bioenergy, v. 29, p. 269-292, 2005.

POLING, B.; PRAUSNITZ, J. M.; O'CONNELL, J. P. Properties of gases and liquids. 5 ed. New York: McGraw-Hill Education, 2001.

RÉMON, J.; GIMÉNEZ, J. R.; VALIENTE, A.; GARCÍA, L.; ARAUZO, J. Production of gaseous and liquid chemicals by aqueous phase reforming of crude glycerol: influence of operating conditions on the process. Energy Conv. Manag., v. 110, p. 90-112, 2016.

SCHWENGBER, C. A.; ALVES, H. J.; SCHAFFNER, R. A.; SILVA, F. A.; SEQUINEL, R.; $\mathrm{BACH}, \mathrm{V}$. R.; FERRACIN, R. J. Overview of glycerol reforming for hydrogen production. Renew. Sust. Energy Rev., v. 58, p. 259-266, 2016.

TANG, H.; KITAGAWA, K. Supercritical water gasification of biomass: thermodynamic analysis with direct Gibbs free energy minimization. Chem. Eng. J., v. 106, p. 261-267, 2005. 